\title{
INVERSE SCATTERING ON THE LINE WITH INCOMPLETE SCATTERING DATA
}

\author{
Tuncay Aktosun \\ Department of Mathematics and Statistics \\ Mississippi State University \\ Mississippi State, MS 39762, USA
}

\begin{abstract}
The Schrödinger equation is considered on the line when the potential is real valued, compactly supported, and square integrable. The nonuniqueness is analyzed in the recovery of such a potential from the data consisting of the ratio of a corresponding reflection coefficient to the transmission coefficient. It is shown that there are a discrete number of potentials corresponding to the data, all those potentials are identified, their $L^{2}$ norms are related to each other in a simple manner, and it is also shown how an additional estimate on the $L^{2}$-norm in the data can uniquely identify the corresponding potential. The recovery is illustrated with some explicit examples.
\end{abstract}

PACS (2003): 02.30.Zz, 03.65.Nk

Mathematics Subject Classification (2000): 34A55, 34L25, 34L40, 47A40, 81U05

Keywords: Schrödinger equation, Inverse scattering, Potential recovery, Incomplete data Short title: Inverse scattering with incomplete data 


\section{INTRODUCTION}

In this paper we analyze the recovery of the potential in the Schrödinger equation on the line from a set of scattering data containing no information on bound states. Our work is motivated by the following question of Paul Sacks: Consider two potentials in the Schrödinger equation where one potential is obtained from the other by adding a bound state. Can we compare the $L^{2}$-norms of these two potentials, and can we conclude that the potential with fewer bound states has a smaller $L^{2}$-norm? By using (2.13) and (2.17) these questions can be answered as follows: Take a square-integrable potential and add a bound state with bound-state energy $-\kappa^{2}$ and any bound-state norming constant. The new potential will have a larger $L^{2}$-norm differing from the previous $L^{2}$-norm by the exact value of $16 \kappa^{3} / 3$. Note that such a difference is independent of the value of the norming constant used, and hence $L^{2}$-norms of square-integrable potentials are affected only by bound-state energies and not by norming constants.

Our work is also motivated by the work of Rundell and Sacks [1], where it was shown that a bounded, real-valued, compactly-supported potential with a sufficiently small $L^{2}$ norm is uniquely determined by the corresponding ratio of a reflection coefficient to the transmission coefficient. With the help of the results in [2], our work here quantifies the smallness of the $L^{2}$-norm in the result of [1]. In Section 3 we present the exact least upper bound for that $L^{2}$-norm, below which we are assured the unique determination of a real-valued, compactly-supported, square-integrable potential in terms of the ratio of a reflection coefficient to the transmission coefficient; we do not require the potential to be bounded.

Let us now establish our notation. We consider the Schrödinger equation

$$
\psi^{\prime \prime}(k, x)+k^{2} \psi(k, x)=V(x) \psi(k, x), \quad x \in \mathbf{R},
$$

where the potential $V$ belongs to the Faddeev class, i.e. it is real valued, measurable, and in $L_{1}^{1}(\mathbf{R})$, the class of measurable functions on the real axis $\mathbf{R}$ such that $\int_{-\infty}^{\infty} d x(1+|x|)|V(x)|$ is finite. The prime is used for the derivative with respect to the spatial coordinate $x$. The Jost solutions $f_{\mathrm{l}}$ and $f_{\mathrm{r}}$, from the left and right, respectively, satisfy the respective boundary conditions

$$
\begin{aligned}
& e^{-i k x} f_{1}(k, x)=1+o(1), \quad e^{-i k x} f_{1}^{\prime}(k, x)=i k+o(1), \quad x \rightarrow+\infty, \\
& e^{i k x} f_{\mathrm{r}}(k, x)=1+o(1), \quad e^{i k x} f_{\mathrm{r}}^{\prime}(k, x)=-i k+o(1), \quad x \rightarrow-\infty,
\end{aligned}
$$


and the transmission coefficient $T$, and the reflection coefficients $L$ and $R$, from the left and right, respectively, are obtained from the spatial asymptotics

$$
\begin{array}{ll}
f_{\mathrm{l}}(k, x)=\frac{e^{i k x}}{T(k)}+\frac{L(k) e^{-i k x}}{T(k)}+o(1), & x \rightarrow-\infty, \\
f_{\mathrm{r}}(k, x)=\frac{e^{-i k x}}{T(k)}+\frac{R(k) e^{i k x}}{T(k)}+o(1), & x \rightarrow+\infty .
\end{array}
$$

A bound state of (1.1) is a square-integrable solution, and such states occur only at the $k$-values on $\mathbf{I}^{+}$in the upper half complex $k$-plane $\mathbf{C}^{+}$where $T(k)$ has (simple) poles. Note that $\mathbf{I}^{+}:=i(0,+\infty)$ denotes the positive imaginary axis. Later we will let $\overline{\mathbf{C}^{+}}:=\mathbf{C}^{+} \cup \mathbf{R}$ and $\mathbf{I}^{-}:=i(-\infty, 0)$. The behavior at $k=0$ tells us whether the potential in (1.1) is generic or exceptional: The generic case occurs if $T(0)=0$ and the exceptional case occurs if $T(0) \neq 0$. For a review of scattering and bound states of $(1.1)$, the reader is referred to [3-9] and the references therein.

Our paper is organized as follows: In Section 2 we briefly review the effect of adding a bound state to a potential and show that certain integrals of the resulting potential remains unaffected by the bound-state norming constant but affected only by the boundstate energy and in a rather simple manner. In Section 3 we analyze a consequence of the result of Section 2 in the recovery of a real-valued, compactly-supported, square-integrable potential in terms of the data $L(k) / T(k)$. We show that, corresponding to that data, there are a discrete number of potentials, and an additional estimate on the $L^{2}$-norm in the data allows the unique identification of a potential among all. We also illustrate the recovery with some explicit examples.

\section{EFFECT OF BOUND STATES ON NORMS OF A POTENTIAL}

Let $V^{[0]}$ denote a potential in the Faddeev class with no bound states. We use $V^{[N]}$ for the potential obtained from $V^{[0]}$ by adding $N$ bound states at $k=i \kappa_{j}$ with the corresponding bound-state dependency constants $\gamma_{j}$, where we have the ordering $0<\kappa_{1}<$ $\cdots<\kappa_{N}$. The superscript $[j]$ refers to quantities associated with the potential $V^{[j]}$; for example, $T^{[j]}, R^{[j]}$, and $L^{[j]}$ denote the scattering coefficients, and $f_{\mathrm{l}}^{[j]}$ and $f_{\mathrm{r}}^{[j]}$ denote the left and right Jost solutions. Recall [4,9] that the dependency constants $\gamma_{j}$ are defined as

$$
\gamma_{j}:=\frac{f_{1}^{[N]}\left(i \kappa_{j}, x\right)}{f_{\mathrm{r}}^{[N]}\left(i \kappa_{j}, x\right)}, \quad 1 \leq j \leq N
$$


and the sign of $\gamma_{j}$ is such that $(-1)^{N-j} \gamma_{j}>0$. It is already known that

$$
\begin{gathered}
T^{[N]}(k)=T^{[0]}(k) \prod_{j=1}^{N} \frac{k+i \kappa_{j}}{k-i \kappa_{j}}, \\
R^{[N]}(k)=(-1)^{N} R^{[0]}(k) \prod_{j=1}^{N} \frac{k+i \kappa_{j}}{k-i \kappa_{j}}, \quad L^{[N]}(k)=(-1)^{N} L^{[0]}(k) \prod_{j=1}^{N} \frac{k+i \kappa_{j}}{k-i \kappa_{j}} .
\end{gathered}
$$

For the known facts listed in this section, we refer the reader to [4], where it is shown that bound states can be added to a potential via the Darboux transformation. We have

$$
V^{[j]}(x)-V^{[j-1]}(x)=-2 \mu_{j}^{\prime}(x), \quad 1 \leq j \leq N,
$$

where we have defined

$$
\mu_{j}(x):=\frac{\chi_{j}^{\prime}(x)}{\chi_{j}(x)}, \quad \chi_{j}(x):=f_{1}^{[j-1]}\left(i \kappa_{j}, x\right)+\left|\gamma_{j}\right| f_{\mathrm{r}}^{[j-1]}\left(i \kappa_{j}, x\right) .
$$

It is known that $\mu_{j}(x)$ is continuous, strictly positive, and differentiable. In fact, as seen from (2.4) we have

$$
\mu_{j}^{\prime}(x)=V^{[j-1]}(x)+\kappa_{j}^{2}-\mu_{j}(x)^{2},
$$

and hence from (2.3) it follows that

$$
V^{[j]}(x)+V^{[j-1]}(x)=2\left[\mu_{j}(x)^{2}-\kappa_{j}^{2}\right]
$$

Define

$$
I_{j, n}(x):=\left[V^{[j]}(x)-V^{[j-1]}(x)\right]\left[V^{[j]}(x)+V^{[j-1]}(x)\right]^{n}, \quad n \geq 0, \quad 1 \leq j \leq N .
$$

Theorem 2.1 Let $V^{[j]}$ be the potential obtained from $V^{[0]}$ by adding bound states of energy $-\kappa_{1}^{2}, \ldots,-\kappa_{j}^{2}$, and assume that $V^{[0]}$ belongs to the Faddeev class without any bound states. We then have

$$
\int_{-\infty}^{\infty} d x I_{j, n}(x)=(-1)^{n+1} 2^{n+2} \kappa_{j}^{2 n+1} \frac{n !}{(2 n+1) ! !}, \quad n \geq 0, \quad 1 \leq j \leq N,
$$

where $(2 n+1) ! !:=(1)(3)(5) \cdots(2 n+1)$.

PROOF: Taking the $n$th power in (2.5) and expanding the result, from (2.3) and (2.5) we get

$$
I_{j, n}(x)=-2^{n+1} \frac{d}{d x} \sum_{p=0}^{n}(-1)^{n-p} \kappa_{j}^{2(n-p)}\left(\begin{array}{c}
n \\
p
\end{array}\right) \frac{\mu_{j}(x)^{2 p+1}}{2 p+1}
$$


where $\left(\begin{array}{c}n \\ p\end{array}\right):=\frac{n !}{p !(n-p) !}$ is the binomial coefficient. It is already known that

$$
\mu_{j}(x)=\left\{\begin{array}{l}
\kappa_{j}+o(1), \quad x \rightarrow+\infty \\
-\kappa_{j}+o(1), \quad x \rightarrow-\infty
\end{array}\right.
$$

Integrating (2.6) on $\mathbf{R}$ and using (2.9), we get

$$
\int_{-\infty}^{\infty} d x I_{j, n}(x)=(-1)^{n+1} 2^{n+2} \kappa_{j}^{2 n+1} \sum_{p=0}^{n}(-1)^{p}\left(\begin{array}{l}
n \\
p
\end{array}\right) \frac{1}{2 p+1} .
$$

Note that the summation in (2.10) can be evaluated explicitly with the help of

$$
\sum_{p=0}^{n}(-1)^{p}\left(\begin{array}{l}
n \\
p
\end{array}\right) \frac{1}{2 p+1}=\int_{0}^{1} d x\left(1-x^{2}\right)^{n}=\frac{n !}{(2 n+1) ! !}, \quad n \geq 0 .
$$

Thus, using (2.11) in (2.10) we establish (2.7).

The result in (2.7) is remarkable in the sense that even though the integrand $I_{j, n}(x)$ depends on the bound-state data $\left\{\kappa_{p}, \gamma_{p}\right\}_{p=1}^{j}$, its integral given in (2.7) is independent of the bound-state data, except for a rather simple $\kappa_{j}$-dependence.

For $n=0$ and $n=1$, respectively, from (2.7) we get

$$
\begin{gathered}
\int_{-\infty}^{\infty} d x\left[V^{[j]}(x)-V^{[j-1]}(x)\right]=-4 \kappa_{j}, \quad 1 \leq j \leq N, \\
\int_{-\infty}^{\infty} d x\left[V^{[j]}(x)^{2}-V^{[j-1]}(x)^{2}\right]=\frac{16}{3} \kappa_{j}^{3}, \quad 1 \leq j \leq N .
\end{gathered}
$$

By summing both sides in each of (2.12) and (2.13) over $j$, we get the following:

Corollary 2.2 Let $V^{[0]}$ be a potential in the Faddeev class with no bound states; add $N$ bound states with energy $-\kappa_{1}^{2}, \ldots,-\kappa_{N}^{2}$, resulting in the potential $V^{[N]}$. We then have

$$
\begin{gathered}
\int_{-\infty}^{\infty} d x\left[V^{[N]}(x)-V^{[0]}(x)\right]=-4 \sum_{j=1}^{N} \kappa_{j}, \\
\int_{-\infty}^{\infty} d x\left[V^{[N]}(x)^{2}-V^{[0]}(x)^{2}\right]=\frac{16}{3} \sum_{j=1}^{N} \kappa_{j}^{3} .
\end{gathered}
$$

Let us indicate some resemblance between the result in (2.7) and the conserved quantities for an evolution equation that is exactly solvable by the inverse scattering transform [12-15]. For example, consider the time-evolution of the scattering data of (1.1) as 
$T(k) \mapsto T(k), L(k) \mapsto L(k) e^{-8 i k^{3} t}, \kappa_{j} \mapsto \kappa_{j}$, and $\gamma_{j} \mapsto \gamma_{j} e^{-8 \kappa_{j}^{3} t}$. The potential of (1.1) then evolves as $V(x) \mapsto u(x, t)$, where $u(x, t)$ satisfies the initial-value problem for the Korteweg-de Vries equation (KdV)

$$
u_{t}-6 u u_{x}+u_{x x x}=0, \quad x \in \mathbf{R}, \quad t>0 ; \quad u(x, 0)=V(x) .
$$

It is known [12-15] that $\int_{-\infty}^{\infty} d x u(x, t), \int_{-\infty}^{\infty} d x u(x, t)^{2}$, and an infinite number of other integrals are independent of $t$ even though their integrands contain $t$ explicitly. Such quantities are known as the conserved quantities for the $\mathrm{KdV}$. Consider now, for example, (2.16) and (2.17), and let us time evolve the potentials $V^{[0]}(x)$ and $V^{[N]}(x)$ to obtain the corresponding solutions $u^{[0]}(x, t)$ and $u^{[N]}(x, t)$ of the KdV. Due to the fact that the bound-state energies $-\kappa_{j}^{2}$ remain unchanged during the time evolution and that the right hand sides in (2.16) and (2.17) do not contain the dependency constants $\gamma_{j}$, we have

$$
\begin{gathered}
\int_{-\infty}^{\infty} d x\left[u^{[N]}(x, t)-u^{[0]}(x, t)\right]=-4 \sum_{j=1}^{N} \kappa_{j}, \\
\int_{-\infty}^{\infty} d x\left[u^{[N]}(x, t)^{2}-u^{[0]}(x, t)^{2}\right]=\frac{16}{3} \sum_{j=1}^{N} \kappa_{j}^{3} .
\end{gathered}
$$

Other similar conserved quantities for the $\mathrm{KdV}$ can be obtained with the help of (2.7).

\section{RECOVERY OF THE POTENTIAL FROM $L(k) / T(k)$}

In [1] the recovery of a bounded, real-valued, compactly-supported potential is considered in terms of the data $\mathcal{D}(k):=L(k) / T(k)$ known for $k \in \mathbf{R}$. In the class of such potentials corresponding to the same $\mathcal{D}(k)$, it was shown (cf. Theorem 2.3 of [1]) that there exists a positive constant $C$ such that if $V_{1}$ and $V_{2}$ are two potentials with $L^{2}$-norms not exceeding $C$ then $V_{1} \equiv V_{2}$. The uniqueness and the reconstruction were obtained by transforming the problem into an equivalent time-domain problem; however, the value of $C$ was left unspecified. In this section, we show how the value of $C$ can be specified.

Recently, we have analyzed [2] the recovery of the potential $V$ of $(1.1)$ from $\mathcal{D}(k)$ when $V$ belongs to the Faddeev class. In this inverse problem, the construction of $V$ is equivalent to the construction of the data $\left\{L(k), N,\left\{\kappa_{j}\right\},\left\{\gamma_{j}\right\}\right\}$, where $L$ is the left reflection coefficient, $N$ is the number of bound states, the set $\left\{-\kappa_{j}^{2}\right\}_{j=1}^{N}$ corresponds to the bound-state energies, and the set $\left\{\gamma_{j}\right\}_{j=1}^{N}$ corresponds to the bound-state dependency constants. We have four cases to consider: 
(a) No information is available on the support of $V$, and the only data available is $\mathcal{D}(k)$.

(b) In addition to $\mathcal{D}(k)$, it is known that the support of $V$ is confined to a half line. In this case, there is no loss of generality in assuming that $V \equiv 0$ for $x<0$.

(c) In addition to $\mathcal{D}(k)$, it is known that the support of $V$ is confined a finite interval. In this case, there is no loss of generality in assuming that $V \equiv 0$ for $x \notin[0,1]$.

(d) In addition to $\mathcal{D}(k)$ and knowledge that $V \equiv 0$ for $x \notin[0,1]$, it is known that $V$ is square integrable and some information related to the $L^{2}$-norm is available. Such additional information may be in the form of a positive constant $C$ which acts as an upper bound on the $L^{2}$-norm.

Let us consider the construction of $V$ or equivalently of $\left\{L(k), N,\left\{\kappa_{j}\right\},\left\{\gamma_{j}\right\}\right\}$ in each of these four cases. For the analysis in the first three cases we refer the reader to [2] and give a brief summary below. Our results show that in case (c), given $\mathcal{D}(k)$ for $k \in \mathbf{R}$, we are able to determine all the corresponding potentials, there are a discrete number of such potentials, the $L^{2}$-norm of each such potential is readily evaluated with the help of (2.17), and appropriate additional information on the $L^{2}$-norm enables us to further restrict the set of potentials corresponding to $\mathcal{D}(k)$. We also explain how the constant $C$ in Theorem 2.3 of [1] arises: that constant allows us to identify the potential with the smallest $L^{2}$-norm among all those corresponding to the same $\mathcal{D}(k)$. By analyzing the inverse problem stated in (d), we show how to determine the precise values of $C$ that can be used in [1].

\section{Case (a): Recovery of $V$ from $\mathcal{D}$ with no Support Information}

If no information other than $\mathcal{D}(k)$ is available, we have the following:

(a.i) If $\mathcal{D}(k)$ is bounded at $k=0$, then there is no restriction on $N$ and hence $N \in$ $\{0,1,2, \ldots\}$. Note that this case corresponds to the exceptional case for (1.1).

(a.ii) If $\mathcal{D}(k)$ is unbounded at $k=0$, then $\lim _{k \rightarrow 0}[2 i k \mathcal{D}(k)]$ is either a positive constant or a negative constant. Thus, either $\mathcal{D}(k) \rightarrow-\infty$ or $\mathcal{D}(k) \rightarrow+\infty$ as $k \rightarrow 0$ on $\mathbf{I}^{+}$. In the former case $N$ must be even, i.e. $N \in\{0,2,4, \ldots\}$; in the latter case $N$ must be odd, i.e. $N \in\{1,3,5, \ldots\}$. Note that both these correspond to the generic case for (1.1).

(a.iii) For each $N$-value resulting from (i) or (ii), given $\mathcal{D}(k)$ there corresponds a $2 N$ parameter family of potentials where the parameter set is $\left\{\kappa_{j}, \gamma_{j}\right\}_{j=1}^{N}$. There are no restrictions on the $\kappa_{j}$ other than $0<\kappa_{1}<\cdots<\kappa_{N}$. There are no restrictions on the $\gamma_{j}$ other than $(-1)^{N-j} \gamma_{j}>0$. 
From the data $\mathcal{D}(k)$ known for $k \in \mathbf{R}$, one uniquely constructs

$$
T^{[0]}(k)=\exp \left(\frac{-1}{2 \pi i} \int_{-\infty}^{\infty} d s \frac{\log \left(1+|\mathcal{D}(s)|^{2}\right)}{s-k-i 0^{+}}\right), \quad k \in \overline{\mathbf{C}^{+}} .
$$

Then, with the help of (2.1) and (2.2), it is seen that the set $\left\{\mathcal{D}(k), N,\left\{\kappa_{j}\right\}\right\}$ leads to the left reflection coefficient given by

$$
L(k)=\mathcal{D}(k) T^{[0]}(k) \prod_{j=1}^{N} \frac{k+i \kappa_{j}}{k-i \kappa_{j}}, \quad k \in \mathbf{R} .
$$

Note that $T^{[0]}(k)$ appearing in (3.1) and (3.2) corresponds to the transmission coefficient for the potential $V^{[0]}$, which is obtained by removing all the $N$ bound states from $V$. The left and right reflection coefficients, $L^{[0]}(k)$ and $R^{[0]}(k)$, respectively, corresponding to $V^{[0]}$ are uniquely determined only in the generic case as

$$
L^{[0]}(k)=(-1)^{N} \mathcal{D}(k) T^{[0]}(k), \quad R^{[0]}(k)=(-1)^{N-1} \mathcal{D}(-k) T^{[0]}(k), \quad k \in \mathbf{R},
$$

because only in the generic case $(-1)^{N}$ is uniquely determined from $\mathcal{D}(k)$. In the exceptional case, the value of $(-1)^{N}$ cannot be determined from $\mathcal{D}(k)$ and hence there are two choices for $V^{[0]}$, which we denote by $V_{1}^{[0]}$ and $V_{2}^{[0]}$, respectively, with the corresponding scattering coefficients determined in terms of $\mathcal{D}(k)$ and $T^{[0]}(k)$ in $(3.1)$ as follows:

$$
\begin{array}{ll}
T_{1}^{[0]}(k)=T^{[0]}(k), & L_{1}^{[0]}(k)=\mathcal{D}(k) T^{[0]}(k), \quad R_{1}^{[0]}(k)=-\mathcal{D}(-k) T^{[0]}(k), \\
T_{2}^{[0]}(k)=T^{[0]}(k), & L_{2}^{[0]}(k)=-\mathcal{D}(k) T^{[0]}(k), \quad R_{2}^{[0]}(k)=\mathcal{D}(-k) T^{[0]}(k) .
\end{array}
$$

For the comparison of two potentials with the same transmission coefficient but with reflection coefficients differing in sign, the reader is referred to $[9,10,11]$. As the next proposition shows, even though $V_{1}^{[0]} \not \equiv V_{2}^{[0]}$, some of their characteristic features are related.

Proposition 3.1 Let $V_{1}^{[0]}$ and $V_{2}^{[0]}$ be two exceptional potentials in the Faddeev class with no bound states, and assume that $T_{1}^{[0]} \equiv T_{2}^{[0]}, L_{1}^{[0]} \equiv-L_{2}^{[0]}$, and $R_{1}^{[0]} \equiv-R_{2}^{[0]}$, i.e. their reflection coefficients differ in sign and their transmission coefficients are the same for all $k \in \mathbf{R}$. Then we have the following:

(i) $\int_{-\infty}^{\infty} d x\left[V_{2}^{[0]}(x)-V_{1}^{[0]}(x)\right]\left[V_{2}^{[0]}(x)+V_{1}^{[0]}(x)\right]^{n}=0, \quad n \geq 0$.

(ii) $V_{1}^{[0]}$ vanishes on a half line if and only if $V_{2}^{[0]}$ vanishes on the same half line. Consequently, $V_{1}^{[0]}$ vanishes outside some interval if and only if $V_{2}^{[0]}$ vanishes outside that interval. 
(iii) If $V_{1}^{[0]}$ vanishes on $\mathbf{R}^{-}$and is continuous on the interval $(0, \delta)$ for some $\delta>0$, then $V_{1}^{[0]}\left(0^{+}\right)=-V_{2}^{[0]}\left(0^{+}\right)$. Similarly, if $V_{1}^{[0]}$ vanishes on $\mathbf{R}^{+}$and is continuous on the interval $(-\delta, 0)$ for some $\delta>0$, then $V_{1}^{[0]}\left(0^{-}\right)=-V_{2}^{[0]}\left(0^{-}\right)$.

PROOF: From (2.24) and (2.25) of [11] we have

$$
V_{2}^{[0]}(x)=V_{1}^{[0]}(x)-2 \rho_{1}^{\prime}(x)=2 \rho_{1}(x)^{2}-V_{1}^{[0]}(x),
$$

where

$$
\rho_{1}(x):=\frac{f_{1 \mathrm{l}}^{[0] \prime}(0, x)}{f_{11}^{[0]}(0, x)}=\frac{f_{1 \mathrm{r}}^{[0] \prime}(0, x)}{f_{1 \mathrm{r}}^{[0]}(0, x)},
$$

with $f_{11}^{[0]}(k, x)$ and $f_{1 \mathrm{r}}^{[0]}(k, x)$ being the left and right Jost solutions for the potential $V_{1}^{[0]}$. It is known that $f_{11}^{[0]}(0, x)$ is continuous and strictly positive and that $\rho_{1}(x)=o(1 / x)$ as $x \rightarrow \pm \infty$. Hence, with the help of (3.4) we get [cf. (2.8)]

$$
\left[V_{2}^{[0]}(x)-V_{1}^{[0]}(x)\right]\left[V_{2}^{[0]}(x)+V_{1}^{[0]}(x)\right]^{n}=-\frac{2^{n+1}}{2 n+1}\left[\rho_{1}(x)^{2 n+1}\right]^{\prime}, \quad n \geq 0,
$$

and integrating both sides over $\mathbf{R}$ we obtain (i). To prove (ii), notice that there is no loss of generality in choosing the half line as $\mathbf{R}^{-}$. If $V_{1}^{[0]} \equiv 0$ for $x<0$, then $f_{1 \mathrm{r}}^{[0]}(0, x)=1$ for $x \leq 0$, and hence $\rho_{1}(x)=0$ for $x \leq 0$. Thus, from (3.4) it follows that $V_{2}^{[0]} \equiv 0$ for $x<0$ as well. Conversely, it follows that $V_{1}^{[0]} \equiv 0$ on $\mathbf{R}^{-}$whenever $V_{2}^{[0]} \equiv 0$ there; thus, we have proved (ii). From (3.4) we see that the first statement in (iii) holds whenever $\rho_{1}\left(0^{+}\right)=0$, which is the case due to the continuity of $\rho_{1}(x)$ at $x=0$ and $\rho_{1}(x)=0$ for $x \leq 0$, which is satisfied when $V_{1}^{[0]} \equiv 0$ for $x<0$. The second statement in (iii) is obtained in a similar manner.

Note that Proposition 3.1(i) holds even when $n$ is a noninteger. By letting $n=0$ and $n=1$ there and using the fact that a potential in the Faddeev class is integrable, we obtain

$$
\int_{-\infty}^{\infty} d x V_{1}^{[0]}(x)=\int_{-\infty}^{\infty} d x V_{2}^{[0]}(x), \quad \int_{-\infty}^{\infty} d x\left[V_{1}^{[0]}(x)^{2}-V_{2}^{[0]}(x)^{2}\right]=0 .
$$

For smooth potentials, we refer the reader to (2.101) of [10] for results similar to (3.5) and their generalizations.

\section{Case (b): Recovery of $V$ from $\mathcal{D}$ with Half-line Support}

If $\mathcal{D}(k)$ is given for $k \in \mathbf{R}$ and if it is also known that $V \equiv 0$ for $x<0$, then, in addition to all the results given in case (a), in particular, in addition to (a.i) and (a.ii), we have the following improvements: 
(b.iii) $\mathcal{D}(k)$ has a unique analytic extension to $k \in \mathbf{C}^{+}$and such an extension is uniquely determined by our data $\mathcal{D}(k)$ known for $k \in \mathbf{R}$. The value of $N$ must satisfy $N \leq$ $Z+1$, where $Z$ denotes the number of zeros of $\mathcal{D}(k)$ on $\mathbf{I}^{+}$. In fact, from the proof of Proposition 3.1 in [2] it follows that if $\mathcal{D}(k)$ has multiple zeros on $\mathbf{I}^{+}$, then $Z$ is actually the number of distinct zeros of odd multiplicity, without counting the multiplicities.

(b.iv) For each $N$-value resulting from restrictions (a.i), (a.ii), and (b.iii), given $\mathcal{D}(k)$ for $k \in \mathbf{R}$, there corresponds an $N$-parameter family of potentials where the parameter set is $\left\{\kappa_{j}\right\}_{j=1}^{N}$. The $\kappa_{j}$ satisfy the restrictions $0<\kappa_{1}<\cdots<\kappa_{N}$ and $(-1)^{N-j} \mathcal{D}\left(i \kappa_{j}\right)>0$ for $j=1, \ldots, N$. The latter restriction confines the $\kappa_{j}$ to subintervals whose endpoints are uniquely determined by the zeros of $\mathcal{D}(k)$ on $\mathbf{I}^{+}$. The dependency constants $\gamma_{j}$ are uniquely determined as $\gamma_{j}=\mathcal{D}\left(i \kappa_{j}\right)$ and hence they are not free parameters. The left reflection coefficient $L(k)$ given in (3.2) becomes meromorphic in $\mathbf{C}^{+}$with simple poles at $k=i \kappa_{j}$ for $j=1, \ldots, N$. Thus, (3.2) now holds for $k \in \overline{\mathbf{C}^{+}}$and the left reflection coefficient $L^{[0]}(k)$ given in (3.3) becomes analytic in $\mathbf{C}^{+}$.

\section{Case (c): Recovery of $V$ from $\mathcal{D}$ with Compact Support}

If $\mathcal{D}(k)$ for $k \in \mathbf{R}$ is given and if it is also known that $V \equiv 0$ for $x \notin[0,1]$, then, in addition to all the results in cases (a) and (b), in particular, in addition to (a.i) and (a.ii), we have the following improvements:

(c.iii) The quantity $k \mathcal{D}(k)$ has a unique analytic extension to the entire complex plane, and such an extension is uniquely determined by our data $\mathcal{D}(k)$ known for $k \in \mathbf{R}$. Moreover, as in (b.iii) the value of $N$ must satisfy $N \leq Z+1$, where $Z$ is the number of zeros of $\mathcal{D}(k)$ on $\mathbf{I}^{+}$having odd multiplicities, without counting the multiplicities.

(c.iv) For each $N$-value resulting from restrictions (a.i), (a.ii), and (c.iii), given $\mathcal{D}(k)$ for $k \in \mathbf{R}$, there correspond a discrete number of potentials where the discrete parameter set is $\left\{\kappa_{j}\right\}_{j=1}^{N}$. The set $\left\{\kappa_{j}\right\}_{j=1}^{N}$ must be a subset of $\left\{\beta_{m}\right\}$ and satisfy the additional restrictions $0<\kappa_{1}<\cdots<\kappa_{N}$ and $(-1)^{N-j} \mathcal{D}\left(i \kappa_{j}\right)>0$ for $j=1, \ldots, N$. Here, each $k=-i \beta_{m}$ corresponds to a zero of $1 / T^{[0]}(k)$ on $\mathbf{I}^{-}$, where $T^{[0]}(k)$ is the quantity in (3.1), and $k / T^{[0]}(k)$ is now entire on $\mathbf{C}$ and uniquely constructed via (3.1) from our data $\mathcal{D}(k)$ known for $k \in \mathbf{R}$. The values $k=-i \beta_{m}$ correspond to the (real) resonances of $V^{[0]}$. For an answer to the question whether the set $\left\{\beta_{m}\right\}$ is a finite set or an infinite set, we refer the reader to [16]. Informally speaking, if $V^{[0]} \in C_{0}^{\infty}[0,1]$ and the order of the zero of $V^{[0]}$ at $x=0$ or at $x=1$ is infinite, then the set $\left\{\beta_{m}\right\}$ may be infinite; 
otherwise, it is a finite set.

\section{Case (d): Recovery of $V$ from $\mathcal{D}$ with Compact Support and $L^{2}$-norm}

Let us assume that $\mathcal{D}(k)$ is given for $k \in \mathbf{R}$ and it is known that $V \equiv 0$ for $x \notin[0,1]$, $V \in L^{2}[0,1]$, and $\|V\| \leq C$, where we denote the $L^{2}$-norm of $V$ as $\|V\|:=\sqrt{\int_{-\infty}^{\infty} d x V(x)^{2}}$. We will determine the precise values of $C$ that assure a unique or nonunique determination of $V$ from $\mathcal{D}$.

As outlined in case (a) below (3.3), given $\mathcal{D}(k)$ for $k \in \mathbf{R}$, we are able to uniquely determine $V^{[0]}$ when $\mathcal{D}$ is singular at $k=0$, and we determine two distinct potentials $V_{1}^{[0]}$ and $V_{2}^{[0]}$ if $\mathcal{D}$ is finite at $k=0$. In the latter case, we know from (3.5) that $\left\|V_{1}^{[0]}\right\|=\left\|V_{2}^{[0]}\right\|$. Thus, $\mathcal{D}(k)$ uniquely determines the $L^{2}$-norm of $V^{[0]}$, even though there are two distinct choices for $V^{[0]}$ in the exceptional case. Let us denote that unique value by $\left\|V^{[0]}\right\|$.

As seen from (c.iv), for each allowed allowed integer $N, \mathcal{D}(k)$ uniquely [2] determines a discrete number of ordered sets $\left\{\kappa_{j}\right\}_{j=1}^{N}$ with the ordering $0<\kappa_{1}<\cdots<\kappa_{N}$ related to the bound states of $V^{[N]}$. Let us define

$$
C_{0}:=\left\|V^{[0]}\right\| ; \quad C_{N}:=\left[\left\|V^{[0]}\right\|^{2}+\frac{16}{3} \sum_{j=1}^{N} \kappa_{j}^{3}\right]^{1 / 2}, \quad 1 \leq j \leq N .
$$

Thus, for each $N, C_{N}$ consists of a sequence of values. Clearly, $C_{0}$ consists of a single number. By listing all the elements in $C_{N}$ for all allowed $N$-values, we obtain a discrete set of ordered positive numbers, which we denote by $\left\{C_{N}\right\}$. This set is a subset of $\left\{\beta_{m}\right\}$, as indicated in (c.iv). The smallest number in the ordered set $\left\{C_{N}\right\}$ is strictly less than the next larger number due to the fact that each set $\left\{\kappa_{j}\right\}_{j=1}^{N}$ with the largest allowable $N$ consists of distinct positive elements. This allows us to determine the value of $C$ in the inequality $\|V\| \leq C$ in order to determine a unique potential $V$ corresponding to our data $\mathcal{D}$. By choosing $C$ as greater than or equal to the smallest number in the set $\left\{C_{N}\right\}$ but strictly less than the next larger element, we will uniquely determine the potential $V$. Next we illustrate this determination with some explicit examples.

As our scattering data let us use $\mathcal{D}(k)=\frac{-\epsilon e^{i k} \sin \sqrt{k^{2}+\epsilon}}{2 i k \sqrt{k^{2}+\epsilon}}$, where $\epsilon$ is a positive parameter. In fact, one corresponding potential is the square well of depth $\epsilon$ supported on the interval $[0,1]$. For each value of $\epsilon$, let us obtain all the potentials corresponding to $\mathcal{D}(k)$ with support confined to $[0,1]$ and specify their $L^{2}$-norms. We have $\lim _{k \rightarrow 0}[2 i k \mathcal{D}(k)]=$ $-\sqrt{\epsilon} \sin \sqrt{\epsilon}$, and hence the exceptional case occurs when $\sqrt{\epsilon} / \pi$ is a positive integer and the 
generic case occurs otherwise. The zeros of $\mathcal{D}(k)$ on $\mathbf{I}^{+}$occur when $\sin \sqrt{k^{2}+\epsilon}=0$, and hence these are all simple zeros occurring at $k=i \sqrt{\epsilon-(j-1)^{2} \pi^{2}}$ for $j=1, \ldots, Z$, with $Z$ being equal to $\lfloor\sqrt{\epsilon} / \pi\rfloor$, i.e. the greatest integer less than or equal to $\sqrt{\epsilon} / \pi$. As $k \rightarrow \infty$ on $\mathbf{I}^{+}$, we have $\mathcal{D}(k) \rightarrow 0^{+}$. As $k \rightarrow 0$ on $\mathbf{I}^{+}$, we get $(-1)^{Z} \mathcal{D}(k) \rightarrow 0^{+}$in the exceptional case, and $(-1)^{Z} \mathcal{D}(k) \rightarrow+\infty$ in the generic case. Define

$$
\frac{1}{\tau(k)}:=e^{i k}\left[\cos \sqrt{k^{2}+\epsilon}+\frac{2 k^{2}+\epsilon}{2 i k \sqrt{k^{2}+\epsilon}} \sin \sqrt{k^{2}+\epsilon}\right] .
$$

Note that $\tau(k)$ corresponds to the transmission coefficient of the square-well potential of depth $\epsilon$ supported on $[0,1]$. It is known that $1 / \tau(k)$ has exactly $Z+1$ (simple) zeros on $\mathbf{I}^{+}$, which we denote by $\xi_{j}$ with the ordering $0<\xi_{1}<\cdots<\xi_{Z+1}$. The quantity in (3.1) is obtained as

$$
\frac{1}{T^{[0]}(k)}=\frac{1}{\tau(k)} \prod_{j=1}^{Z+1} \frac{k+i \xi_{j}}{k-i \xi_{j}}
$$

Example 3.2 When $\epsilon=5$, we are in the generic case and $Z=0$. Hence, $N \leq 1$, but $\mathcal{D}(k) \rightarrow+\infty$ on $\mathbf{I}^{+}$indicates that $N$ must be odd. Thus, $N=1$ is the only allowed value. In this case, $1 / T^{[0]}(k)$ given in (3.6) has two zeros on $\mathbf{I}^{-}$at $k=-i \beta_{j}$ with $\beta_{1}=1.5433 \overline{4}$ and $\beta_{2}=1.585 \overline{7}$. We use an overline to indicate roundoff. In (3.6) we have $\xi_{1}=\beta_{2}$. Corresponding to $\mathcal{D}(k)$ we have two potentials $V_{1}^{[1]}$ and $V_{2}^{[1]}$, having bound states at $k=i \beta_{1}$ and $k=i \beta_{2}$, respectively. Note that $V_{2}^{[1]}$ is the square well of depth $\epsilon$. We have $\left\|V_{1}^{[1]}\right\|=4.8312 \overline{6}$ and $\left\|V_{2}^{[1]}\right\|=5$. Thus, knowledge of any $C$ satisfying $\left\|V_{1}^{[1]}\right\| \leq C<$ $\left\|V_{2}^{[1]}\right\|$ helps us to identify $V_{1}^{[1]}$ or $V_{2}^{[1]}$ as the unique potential corresponding to $\mathcal{D}(k)$. The left reflection coefficients $L_{1}^{[1]}$ and $L_{2}^{[1]}$ corresponding to $V_{1}^{[1]}$ and $V_{2}^{[1]}$, respectively, are obtained from (3.2) as

$$
L_{j}^{[1]}(k)=\mathcal{D}(k) T^{[0]}(k) \frac{k+i \beta_{j}}{k-i \beta_{j}}, \quad j=1,2 .
$$

Example 3.3 When $\epsilon=\pi^{2}$, we are in the exceptional case and $Z=0$. Hence, both $N=0$ and $N=1$ are allowed. In this case $1 / T^{[0]}(k)$ given in (4.1) has only one zero on $\mathbf{I}^{-}$at $k=-i \beta_{1}$ with $\beta_{1}=2.52258 \overline{8}$. In (4.1) we have $\xi_{1}=\beta_{1}$. Corresponding to $\mathcal{D}(k)$ we have two potentials $V^{[0]}$ and $V^{[1]}$, the former with no bound states and the latter with one bound state at $k=i \beta_{1}$. Note that $V^{[1]}$ is the square well of depth $\epsilon$. We have $\left\|V^{[0]}\right\|=3.3853 \overline{7}$ and $\left\|V^{[1]}\right\|=\pi^{2}$. Thus, knowledge of any $C$ satisfying $\left\|V^{[0]}\right\| \leq C<\left\|V^{[1]}\right\|$ helps us to identify either $V^{[0]}$ or $V^{[1]}$ as the unique potential corresponding to $\mathcal{D}(k)$. The left reflection 
coefficients $L^{[0]}$ and $L^{[1]}$ corresponding to $V^{[0]}$ and $V^{[1]}$, respectively, are obtained from (3.2) as

$$
L^{[0]}(k)=\mathcal{D}(k) T^{[0]}(k), \quad L^{[1]}(k)=\mathcal{D}(k) T^{[0]}(k) \frac{k+i \beta_{1}}{k-i \beta_{1}}
$$

Example 3.4 When $\epsilon=20$, we are in the generic case and $Z=1$. Hence, $N \leq 2$, but $\mathcal{D}(k) \rightarrow-\infty$ on $\mathbf{I}^{+}$indicates that $N$ must be even. Thus, $N=0$ and $N=2$ are the only possibilities. In this case $1 / T^{[0]}(k)$ given in (4.1) has two zeros on $\mathbf{I}^{-}$at $k=-i \beta_{1}$ with $\beta_{1}=\xi_{1}=1.9302 \overline{1}$ and $k=-i \beta_{2}$ with $\beta_{2}=\xi_{2}=3.9255 \overline{6}$, where $\xi_{1}$ and $\xi_{2}$ are the quantities in (3.6). When $N=2$, the only potential $V^{[2]}$ corresponding to $\mathcal{D}(k)$ is the square well of depth $\epsilon$ with support $[0,1]$. When $N=0$, the corresponding potential $V^{[0]}$ is uniquely determined from $\mathcal{D}(k)$ and its left reflection coefficient $L^{[0]}(k)$ is obtained from (3.2) as

$$
L^{[0]}(k)=\mathcal{D}(k) T^{[0]}(k) \frac{\left(k-i \beta_{1}\right)\left(k-i \beta_{2}\right)}{\left(k+i \beta_{1}\right)\left(k+i \beta_{2}\right)} .
$$

In this case we have $\left\|V^{[0]}\right\|=6.2463 \overline{5}$ and $\left\|V^{[2]}\right\|=20$. Thus, an appropriate specification of the upper limit on the $L^{2}$-norm of the potential allows the unique identification of $V^{[0]}$ or $V^{[2]}$ from $\mathcal{D}(k)$.

Example 3.5 When $\epsilon=130$, the allowed values for $N$ are 0,2 , and 4 . In this case $1 / T^{[0]}(k)$ given in (4.1) has six zeros on $\mathbf{I}^{-}$at $k=-i \beta_{j}$ with $\beta_{1}=4.8729 \overline{5}, \beta_{2}=8.2260 \overline{7}$, $\beta_{3}=8.3286 \overline{5}, \beta_{4}=10.087 \overline{9}, \beta_{5}=10.740 \overline{7}, \beta_{6}=11.08 \overline{5}$. For $N=0$, the only potential corresponding to $\mathcal{D}(k)$ has norm $\left\|V^{[0]}\right\|=23.96 \overline{8}$. For $N=2$, there are five potentials corresponding to $\mathcal{D}(k)$ with norms $\left\|V_{1}^{[2]}\right\|=64.50 \overline{9},\left\|V_{2}^{[2]}\right\|=65.366 \overline{8},\left\|V_{3}^{[2]}\right\|=91.956 \overline{6}$, $\left\|V_{4}^{[2]}\right\|=115.38 \overline{7},\left\|V_{5}^{[2]}\right\|=120.19 \overline{7}$, where $V_{1}^{[2]}$ has bound states $\left\{-\beta_{1}^{2},-\beta_{2}^{2}\right\}, V_{2}^{[2]}$ has $\left\{-\beta_{1}^{2},-\beta_{3}^{2}\right\}, V_{3}^{[2]}$ has $\left\{-\beta_{1}^{2},-\beta_{6}^{2}\right\}, V_{4}^{[2]}$ has $\left\{-\beta_{4}^{2},-\beta_{6}^{2}\right\}$, and $V_{5}^{[2]}$ has $\left\{-\beta_{5}^{2},-\beta_{6}^{2}\right\}$. For $N=4$, there are four potentials corresponding to $\mathcal{D}(k)$ with norms $\left\|V_{1}^{[4]}\right\|=130$, $\left\|V_{2}^{[4]}\right\|=130.43 \overline{2},\left\|V_{3}^{[4]}\right\|=134.28 \overline{7},\left\|V_{4}^{[4]}\right\|=134.70 \overline{5}$, where $V_{1}^{[4]}$ has bound states $\left\{-\beta_{1}^{2},-\beta_{2}^{2},-\beta_{4}^{2},-\beta_{6}^{2}\right\}, V_{2}^{[4]}$ has $\left\{-\beta_{1}^{2},-\beta_{3}^{2},-\beta_{4}^{2},-\beta_{6}^{2}\right\}, V_{3}^{[4]}$ has $\left\{-\beta_{1}^{2},-\beta_{2}^{2},-\beta_{5}^{2},-\beta_{6}^{2}\right\}$, $V_{4}^{[4]}$ has $\left\{-\beta_{1}^{2},-\beta_{3}^{2},-\beta_{5}^{2},-\beta_{6}^{2}\right\}$. Thus, some appropriate knowledge on the $L^{2}$-norm of the potential allows us to pick a unique potential among all these 16 potentials corresponding to the same $\mathcal{D}(k)$. Note that $V_{1}^{[4]}$ is the square well of depth $\epsilon$.

Acknowledgments. The research leading to this article was supported in part by the National Science Foundation under grant DMS-0204437 and the Department of Energy under grant DE-FG02-01ER45951. 


\section{REFERENCES}

[1] W. Rundell and P. Sacks, On the determination of potentials without bound state data, J. Comput. Appl. Math. 55, 325-347 (1994).

[2] T. Aktosun and V. Papanicolaou, Recovery of a potential from the ratio of reflection and transmission coefficients, J. Math. Phys., to appear in November 2003.

[3] L. D. Faddeev, Properties of the S-matrix of the one-dimensional Schrödinger equation, Amer. Math. Soc. Transl. (Ser. 2) 65, 139-166 (1967).

[4] P. Deift and E. Trubowitz, Inverse scattering on the line, Comm. Pure Appl. Math. 32, 121-251 (1979).

[5] R. G. Newton, The Marchenko and Gel'fand-Levitan methods in the inverse scattering problem in one and three dimensions, In: J. B. Bednar et al. (eds), Conference on inverse scattering: theory and application, SIAM, Philadelphia, 1983, pp. 1-74.

[6] A. Melin, Operator methods for inverse scattering on the real line, Comm. Partial Differential Equations 10, 677-766 (1985).

[7] V. A. Marchenko, Sturm-Liouville operators and applications, Birkhäuser, Basel, 1986.

[8] K. Chadan and P. C. Sabatier, Inverse problems in quantum scattering theory, 2nd ed., Springer, New York, 1989.

[9] T. Aktosun and M. Klaus, Chapter 2.2.4, Inverse theory: problem on the line, In: E. R. Pike and P. C. Sabatier (eds.), Scattering, Academic Press, London, 2001, pp. $770-785$.

[10] A. Degasperis and P. C. Sabatier, Extension of the one-dimensional scattering theory, and ambiguities, Inverse Problems 3, 73-109 (1987).

[11] T. Aktosun, M. Klaus, and C. van der Mee, On the Riemann-Hilbert problem for the one-dimensional Schrödinger equation, J. Math. Phys. 34, 2651-2690 (1993).

[12] P. D. Lax, Integrals of nonlinear equations of evolution and solitary waves, Comm. Pure Appl. Math. 21, 467-490 (1968).

[13] G. L. Lamb, Jr., Elements of soliton theory, Wiley, New York, 1980.

[14] M. J. Ablowitz and H. Segur, Solitons and the inverse scattering transform, SIAM, Philadelphia, 1981.

[15] M. J. Ablowitz and P. A. Clarkson, Solitons, nonlinear evolution equations and inverse scattering, Cambridge University Press, Cambridge, 1991.

[16] M. Zworski, Distribution of poles for scattering on the real line, J. Funct. Anal. 73, 277-296 (1987). 